\title{
The presence of occupational structure in online texts based on word embedding NLP models
}

\author{
Zoltán Kmetty ${ }^{1,2}$, Júlia Koltai ${ }^{1,23^{*}}$ (D) and Tamás Rudas ${ }^{2}$
}

\footnotetext{
*Correspondence: koltai.julia@tk.hu ${ }^{1}$ CSS-Recens Research Group, Centre for Social Sciences Hungarian Academy of Sciences Centre of Excellence, Tóth Kálmán u. 4, 1097, Budapest, Hungary ${ }^{2}$ Faculty of Social Sciences, Eötvös Loránd University, Pázmány Péter sétány 1/A, 1117, Budapest, Hungary

Full list of author information is available at the end of the article
}

\begin{abstract}
Research on social stratification is closely linked to analyzing the prestige associated with different occupations. This research focuses on the positions of occupations in the semantic space represented by large amounts of textual data. The results are compared to standard results in social stratification to see whether the classical results are reproduced and if additional insights can be gained into the social positions of occupations. The paper gives an affirmative answer to both questions.

The results show a fundamental similarity of the occupational structure obtained from text analysis to the structure described by prestige and social distance scales. While our research reinforces many theories and empirical findings of the traditional body of literature on social stratification and, in particular, occupational hierarchy, it pointed to the importance of a factor not discussed in the mainline of stratification literature so far: the power and organizational aspect.
\end{abstract}

Keywords: Social stratification; Prestige; Occupations; Natural Language Processing; Word embedding; Text mining

\section{Introduction}

Analysis and positioning of occupations in social space have a long history in social research. Social stratification models use occupations as a standard way of operationalizing the position of people in society. Most of the stratification models rely on massive survey data. However, the developments of information technology, particularly data science and natural language processing (NLP), and the rapid growth of computing capacity provide new types of data sources. NLP methods-like word embedding used in this analysisopen up the opportunity to examine society through written/digitalized texts.

The language used by a social group mirrors the group's cultural frame of mind (Kozlowski et al. [21]). These texts inform us about the ways of thinking, feeling, and knowledge of people. (Evans-Aceves [9]). Billions of digitalized or originally digital texts are available for analysis, depicting mentality, opinion, and values. Sources of texts vary from social media posts, through online newspapers and forums, to whole books of classic literature or scientific papers. Thus, analyzing these vast corpora can help understand people's perceptions and ways of thinking in a given culture about any topic.

(c) The Author(s) 2021. This article is licensed under a Creative Commons Attribution 4.0 International License, which permits use sharing, adaptation, distribution and reproduction in any medium or format, as long as you give appropriate credit to the original author(s) and the source, provide a link to the Creative Commons licence, and indicate if changes were made. The images or other third party material in this article are included in the article's Creative Commons licence, unless indicated otherwise in a credit line to the material. If material is not included in the article's Creative Commons licence and your intended use is not permitted by statutory regulation or exceeds the permitted use, you will need to obtain permission directly from the copyright holder. To view a copy of this licence, visit http://creativecommons.org/licenses/by/4.0/. 
Our paper focuses on the positions of occupations in the semantic space represented by large amounts of textual data. The results are compared to standard results in social stratification to see whether the classical results are reproduced and if additional insights can be gained into the social positions of occupations. The paper gives an affirmative answer to both questions.

The main contribution of this paper is that social structures, in particular, stratification of occupations-established so far based on purposively collected data-exist and can be derived from large text corpora using methods of unsupervised learning. Further, the most critical factors organizing this stratification can be implied, not from theoretical considerations, instead of the semantic space depicted in the text corpora.

In the first part of the paper, we briefly introduce a review of how social scientists measure people's position in society. We also discuss NLP basics, especially word embedding models, and give a short review of how occupations have been analyzed using NLP methods. In the Data and Methods chapter, we describe the large digitalized corpora we have used in the analysis and the model's specification, with which we have extracted the latent dimensions of occupations from these corpora. The analysis and the results follow this part. The paper closes with a discussion of how these findings reinforce and extend our understanding of the societal positions of occupations.

\section{Theoretical background}

\subsection{Occupations and social structure}

Social class and social stratification are widely used concepts from the early years of sociology. Some variants of these concepts are theory-driven; others rely on empirical data. Some use categories to describe people's positions in the social structure; others apply continuous scales. In social stratification research, occupation is routinely used to link the positions of the individuals to their memberships in a social stratum. In industrialized societies, occupation is a powerful indicator of social standing. As it tends to be more stable than income, it serves as a much better proxy for the position of an individual (Connelly et al. [6]). Thus, the goal of these researches is to classify the occupations to mirror society's stratification. Multiple approaches exist regarding the measurement of occupational position in social space. Some theories use occupation to create vertical hierarchies with continuous scales (Ganzeboom-Treiman [12]); others use it to develop discrete stratification categories with horizontal and vertical dimensions (Goldthorpe et al. [15], RoseHarrison [30]). Researchers used various measurements for the classification of occupations to create their stratification models. Based on Bukodi et al. [3], we can divide these measurements into two types: one type uses subjective indicators and the other works with objective indicators. The scale of Goldthorpe and Hope [14] belongs to the former category. They applied a synthetic scale of subjective opinions to measure the general desirability of occupations.

Treiman [33] also used questions on subjective perceptions, and from these, he created the Standard International Occupational Prestige Scale (SIOPS), a widely used analytical scale. The International Socio-Economic Index (ISEI) (Ganzeboom-Treiman [12]) and the Cambridge scale (Prandy-Lambert [28], and Meraviglia et al. [23]) are good examples for the other type of scales, which use objective data in their measurement. ISEI builds on the educational level and average income of the occupations to create their hierarchy. The Cambridge scale uses the marriage-table-based social distance of occupations to map their 
order. Chan and Goldthorpe [5] applied a similar methodology, built on close friendship data, not marriage tables. In their interpretation, the scale measures the hierarchy of social status. Meraviglia and her colleagues [23] argue that all continuous measures of social stratification are indicators of the exact latent dimensions.

But which characteristics of the occupations matter? The answer varies from one social stratification model to the other. The Erikson-Goldthorpe-Portocarero (Erikson et al. [8]) (EGP) model-one of the well-known occupation-based stratification models-is built on the employment relations in the labor market. The market and the work situation (e.g., level of income, economic security, authority level) are the dimensions, which determine the class position. (Connelly et al. [6]) Along with education, income also plays an essential role in the construction of the ISEI scale. In the SIOPS scale, occupations are ordered by their prestige, measured by the subjective judgment of respondents of large-scale surveys.

In this paper, we explore how occupation structure could be measured through online texts. Our approach is data-driven, as we unfold the different layers of occupational structure in online digitalized texts, not on purposively collected data. From this viewpoint, the closest model from the abovementioned ones is the Cambridge Scale. However, we do not focus on the social ties but rather on the semantic relations of the occupations. In the following subchapter, we introduce those novel text mining techniques, with which we can examine the semantic ties of the occupations and, through these, study the structure of the society.

\subsection{Text as data and word embedding models}

The process produced data, like text messages, phone calls, public transport usage with digital tickets, social media posts, and bank transfers, all leave digital marks in databases of different systems. These data are not generated by the users with the understanding that they will be part of some analyses; thus, these data mirror the behavior of individuals better than data from classical surveys or other research. While self-reported responses can be biased by the interview situation, social desirability, and limitations to recall past events (Lazer and Radford [22]). For that very reason, the analyses of digital data can be exceptionally interesting for social research.

This information is stored in very diverse formats, from pictures, videos, or voices, to numbers and the majority of these data are stored or can be transformed into textual forms. Text analysis has always had an essential place in the field of sociology. From the line-by-line reading and analysis at the birth of the science, through coding and linking the text by the researcher (Bales [1]) to digital and partly automatized coding of smaller corpora (Hays [17]), it was always part of sociology - which, according to Savage and Burrows [31], defined its expertise through its own methods. However, these classic analytical methods could not handle large-scale corpora with thousands of millions of words. The methodological knowledge needed to analyze large text data had to be imported from computational linguistics, data- and computer science. Parallel with the increase of digital data, computational power, and artificial intelligence have also developed. New methods, which aim at the processing of large digital corpora, emerge and are continuously elaborated. These methods have to be incorporated by sociologists; otherwise, they would miss the opportunity to interpret such data sources (Németh and Koltai [26]).

Just like partly automatized methods of earlier times, automated text analysis and natural language processing combine qualitative and quantitative approaches. The latest practices provide the deepness of qualitative analysis with the advantage of many observations 
in quantitative analysis. However, one of the consequences that these textual data mostly record observed behavior is that its structure and relevance (its 'noisiness') are not as appropriate for analyses as data collected by traditional techniques. The phase of data cleaning and structuring includes important decisions of the researcher. These decisions can influence the inner and outer validity of the results; thus, the very detailed documentation and the description of the arguments behind these decisions are vital for making the research transparent.

Simpler methods of text analysis only focus on the words of the corpus, as if they had no relations with the surrounding words and sentences, but more complex processes can also take the structure of the text into account. Some of these methods are based on the 'bag-of-words model, which means that words are treated together with their environments, namely a given number of words around them. The size (the number of words) of the environment is defined by the researcher and can be any positive integer, though too wide environments can cause loss of context. The examination of the environment has proceeded for each word as a sliding window through the whole corpus, and the result of the method is based on the complex co-occurrences of words.

Our analysis method is a neural network-based word embedding model (Mikolov et al. [24]). This method helps the researcher to understand the deeper meaning of texts by modeling the semantic meaning of words. A word's position is defined by its context, which approach has a non-computerized linguistic theoretical base, originated by Firth [10]. The word embedding model projects the position of each word of a corpus to a low dimensional vector space. The most popular method, Word2Vec (Mikolov et al. [24]), uses a neural network based logistic classifier to estimate the word positions. The corpus words are positioned in this semantical vector space, where we can calculate the contextual proximity of words. This proximity not only replies to the co-occurrences of the words but also the co-occurrences of the contexts of words. Several word embedding methods are available (e.g., Word2Vec by Mikolov et al. [24], Glove by Pennington et al. [27], and Fasttext by Joulin et al. [20]) to train textual data and to establish proximities. ${ }^{1}$ In either method, the positions of a word define its meaning in the semantic space. Two words with similar environments will be close to each other; thus, words with similar meanings will be nearby. Proximities of words are frequently defined by the cosine of the angle formed by the vector of the words. Standard metrics like Euclidean distance could be misleading here because the length of each word vector strongly correlates with the word's frequency within the corpora (and it also depends on the context variability) (Schakel-Wilson [32]).

Kozlowski et al. [21] showed that these proximities could be successfully used to analyze culture. The starting point of their analyses was based on the theoretical foundation that language (and texts) mirrors the way of thinking of those who use them. Thus, the study of written texts allows researchers to conclude the society the texts originate from. They showed that word embedding methods could create dimensions of social inequality with the proximity of words, representing the two extreme values of a given inequality (e.g., poor-rich; male-female). Mirroring this proximity to other words, we can unfold hidden inequalities of the society. For example, if we reflect the dimension of gender to the word

\footnotetext{
${ }^{1}$ The latest generation of word embedding models (like BERT-Devlin et. al [7]) creates contextualized vector spaces and not static ones. They calculate unique word distances for different contexts. These models perform very well in classification tasks, but they are not applicable to analyzes such as ours, which do not look for varying positions of a word in different contexts but targets the general position of words.
} 
'doctor,' we find the word 'nurse' at the other end of the dimension. The gender inequality of these medical professions can be captured in the vector space. (See similar results of Bolukbasi et al. [2], Caliskan et al. [4], and Garg et al. [13]) For sociologists, these analogies can help a lot in the understanding of social cleavages, as based on the concept of Kozlowski et al. [21], they can unfold unconscious or not yet proved patterns of social inequalities.

\section{Data and methods}

In the previous section, we presented the basics of word embedding models and showed how these models could be used to analyze social phenomena. In this research, we use pretrained word vector models. These widely used word vectors are publicly available, which makes our results reproducible. The embeddings are trained on large-scale corpora, which is important as previous research showed that the accuracy and validity of word embedding (measured on word analogies) highly depend on the corpus size (Mikolov [24]). These pre-trained vector spaces are frequently used in NLP tasks. But previous studies have also confirmed that these vector space models can be used well to study social processes and social context as well. Researchers have validated with surveys that vector space models trained on large and general corpus can be used to measure cultural patterns (Kozlowski et al. [21]) or even stereotypes against social groups (Joseph-Morgan [19]).

We used three pre-trained vector spaces in the analysis. The first vector model we used was trained on the English language texts of the Common Crawl (CC) corpus, ${ }^{2}$ a huge web archive, which contains raw web page data, metadata and text extractions. The raw web pages can be everything, from a news site, blog, or university page, to pages like Amazon Books. As the authors state, they provide "a copy of the internet." It consists of one petabyte of data collected between 2011 and 2017. The word embedding model was trained on the English language pages of this corpus. As the data do not contain geolocation of the websites, they might include websites worldwide. In the initial corpus, 600 billion tokens were identified, and the vector space consists of 2 million words positioned in a 300-dimensional space. ${ }^{3}$ The training of the corpus was realized by Fasttext algorithm (Joulin et al. [20]).

The second vector space we used is the Wikinews, trained on a combined corpus of the English Wikipedia (saved in 2017), the UMBC WebBase corpus, and another corpus, which contains all the news from stamt.org. The UMBC corpus contains high-quality English paragraphs derived from the Stanford WebBase project and includes 100 million web pages from 2007. Statmt.org contains political and economic commentary crawled from the website Project Syndicate. The combined corpus is quite diverse and has 16 billion tokens. The vector space consists of one million words, positioned in 300-dimensional space, and was trained by the Fasttext algorithm (Joulin et al. [20]). Thus, the number of dimensions and the training method of the two vector spaces were the same.

We used a third vector space, which was also built on a combined corpus of the Wikinews sources, but in this third vector space, sub-word information was also taken into account during the training phase of the model. It means that partly identical or words or words with the same root like sociology and society tend to be closer to each other in this

\footnotetext{
${ }^{2}$ http://commoncrawl.org

${ }^{3}$ https://fasttext.cc/docs/en/english-vectors.html
} 


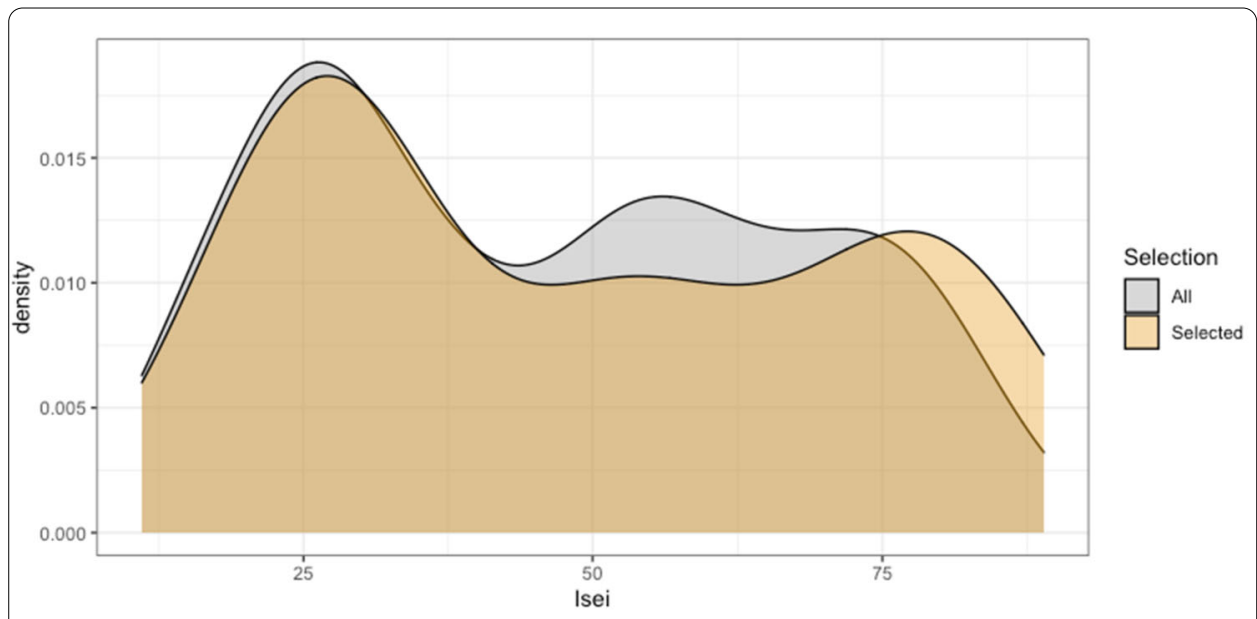

Figure 1 Kernel density histogram of ISEI scores of all categories and selected categories

vector space. We will refer to this vector space later as Wikinews Subwords. On this vector space, we utilize the innovation of the Fasttext algorithm, namely that it can account for sub-word information. Although the first two vector spaces were also trained by the Fasttext algorithm, sub-word information was not taken into account there. Thus, the method was closer to a word2vec solution (which cannot handle subword information).

The pre-trained vectors we used in this paper are trained on general English corpora. We could not narrow the geographical focus, as we do not know the geographical distribution of the authors of texts. However, based on other results in this topic (see Treimann [33]), there are no significant differences between the prestige scores of different developed countries.

Altogether 234 occupations (see Section A.1 for the list) were selected for the analysis, and we used the most common 200,000 words of each vector space. In the ISCO classification, more than 7000 occupations are listed, but the number of one-world-length occupations was around 750. We manually checked all these occupations and selected those more than 200, which were not extra unique or rare (like chieftain). These occupations cover both the vertical and horizontal aspects of occupational space. Although we tried to create a gender-balanced occupational list, male occupations are overrepresented based on our qualitative estimations. From the 410 ISCO categories, we selected 129 in the analysis. The ISEI range was similar in the selected occupations to those in the complete list of occupations; however, the mean value was slightly higher in the selected list (48 vs. 46).

The kernel density histogram (see Fig. 1) shows the high similarity of the selected occupations with the complete list. It also points out a slightly higher representation of higher ISEI score occupations, but as it is presented, the difference is negligible and cannot bias the analysis. Some of the pre-selected occupations were not among the most common 200,000 words, so we had to omit them. In the end, from these 234 occupations, 204 occupations were detected in CC and 207 in Wikinews (202 occupations were available in both corpora). We located the position of each 204 and 207 occupations in the vector spaces. The same methods were applied for each vector space (CC, Wikinews. Wikinews Subwords): the cosine-similarities of each pair of occupations were computed in the 300dimensional vector space. These cosine-similarities are the ones, which represent the se- 
Table 1 Semantic closeness of selected occupations (cosine similarity, CC corpus)

\begin{tabular}{|c|c|c|c|c|c|c|}
\hline & Doctor & Cardiologist & Sociologist & Historian & Shopkeeper & Barmaid \\
\hline Doctor & 1.00 & 0.61 & 0.29 & 0.25 & 0.34 & 0.25 \\
\hline Cardiologist & & 1.00 & 0.32 & 0.27 & 0.20 & 0.11 \\
\hline Sociologist & & & 1.00 & 0.62 & 0.31 & 0.25 \\
\hline Historian & & & & 1.00 & 0.26 & 0.20 \\
\hline Shopkeeper & & & & & 1.00 & 0.48 \\
\hline Barmaid & & & & & & 1.00 \\
\hline
\end{tabular}

mantic closeness of the occupations. Table 1 shows a small part of the similarity table based on the CC corpus.

As we only dealt with similar concepts, namely occupations, most words have positive cosine similarity: the mean value was 0.25 across all pairs of occupations. (The theoretical range of cosine similarity is between -1 and 1.)

Over this similarity, we can observe significant differences in the values of the table. Not surprisingly, the occupation doctor is close to the cardiologist, sociologist is close to the historian, and the shopkeeper is close to the barmaid. At the same time, the doctor is distant from sociologists, historians, and barmaid, and the shopkeeper is distant from cardiologists and historians. We can observe that distinct domain areas of occupations can be identified based on the similarity matrix. In Table 1, only positive values are present, but negative cosine similarity is also possible. The lowest value in the CC corpus was -0.02 between cleaner and rheumatologist.

As we have mentioned in the Introduction, one of the main goals of our research was to extract the most critical dimensions, which structure the occupations in the semantic field, and to see whether we can find similar latent structures behind the occupations like the one of ISEI. Therefore, dimension reduction methods were at the center of our interest. We applied factor analysis with rotation of the similarity matrix-instead of the often used correlation matrix - as input, ${ }^{4}$ as based on the literature, we assumed that more than one dimension of occupation ranking exists. Although Principal Component Analysis (PCA) could also have been a possible approach, PCA (theoretically) is more beneficial for pure dimension reduction, while factor analysis is efficient in finding latent structures.

However, to test the robustness of the results, we also run a PCA model. Without rotation, the first component showed the centrality of the occupations, so those occupations with high mean similarities with other professions had high scores in the first PCA (the Pearson correlation coefficient between centrality and PCA score was 0.99 ). When we rotated the PCA (with varimax rotation), we obtained similar results to the factor models.

The presented results are based on a minres (Minimum Residual) factor analysis technique and varimax rotation (Revelle [29]). As further robustness tests, we repeated our computations with different factor analysis methods and rotation techniques; the differences between these and the initial results were relatively small. For example, we had the same results when we applied an ML factor analysis and Equamax rotation as we got with minres and varimax rotation earlier.

\footnotetext{
${ }^{4}$ Most scientific papers using word embedding models use cosine similarity to calculate the distance between words, and above in the paper, we also argue for this choice, compared to, for example, Euclidean distance. On the other hand, correlation can also be a good choice, and it is not even far from cosine similarity: Pearson correlation is, in fact, a centered Cosine similarity. Cosine similarity values in the distance matrix are very similar to correlation values: the maximum difference was 0.02 between them. Regarding these results, both cosine similarity and correlation can be applied to measure the closeness of the occupations, as they provide very similar results. We stuck to cosine similarity because in the case of word embedding applications, cosine similarity is the standard measurement.
} 
Due to the exploratory nature of the research, we have not had a strong assumption on the number of factors to be extracted. The decision on the number of factors was based on empirical tests and also on practical considerations. We decided to select more than 1 factor as we wanted to understand the most critical dimensions behind the structure of the occupations and not only the primary dimension. At the same time, we decided to select a maximum of 5 factors to keep the interpretability. Average residuals for the similarity matrix (RMSR value) and Chi-square-based fit indices were used to test the statistical validity of the models, and external measures (like the ISEI scale) were applied for the comparison of the results to test criterion validity. Overall, we found that all the $2,3,4$, and 5-factor solutions are worth investigating. In the later analysis, we detail the 3-factor solution as it looked the most promising one.

We used different methods for the robustness test of the models. We compared the consistency of the results of varying vector spaces with the cross-correlation of the factors generated in the different vector spaces. We also tested the similarity of the context of the exact words across different vector spaces. Suppose we have two independently trained vector spaces. In that case, the cosines similarity of the exact words in the different vector spaces is around 0 , as the position of the words is doubtful to be the same in the two vector spaces. Thus, to compare the context of the words, first, we have to align the two vector spaces. To test the context similarity of the words across different vector spaces, from the most frequent 200,000 words of each corpus, we selected those 153423 words that appear in both corpora. We aligned the Wikinews vector space to the Common Crawl vector space with Procrustes rotation. In the aligned Wikinews vector space, the cosine similarity of occupation pairs remained the same, but we could calculate the similarity of the exact words in the two embeddings. In a case of perfect alignment, the cosine similarity would be 1 . But in real-world examples, the similarity never reaches the celling point (1), as the training process adds some random variation and also because the context of the words is different. But higher similarity means higher context stability across embeddings. This alignment technique has been used in previous papers to measure the context variation of other concepts through time (Hamilton-Leskovec-Jurafsky [16]). Still, in this paper, we primarily use this to measure the stability of occupation contexts across embeddings based on different text corpora.

\section{Heuristics}

Before starting the analysis of occupations in the vector spaces, we present some examples about the context of occupations to show it more intuitively what these models are based on. The goal is to measure social structure through the semantic position of occupation. The most important question is how social structure is presented in textual data. In the examples below, we selected some sentences, which include occupations. Here we use cultural examples to explain the social position of occupations. But we could replace the cultural examples with any other life domain. We ask the reader of this paper to go through these examples and think about if it is possible to change the occupations between the sentences and the likelihood that the revised sentence will occur.

\section{Example 1}

Last night the SENATOR went to the theatre

This evening the TYPIST wanted to go bowling. 
Table 2 cosine similarity and ISEI distance of occupation pairs (example)

\begin{tabular}{lll}
\hline & Cosine similarity in the CC corpus & ISEl distance \\
\hline Anatomist-ornithologist & 0.49 & 0 \\
Barman-bartender & 0.81 & 0 \\
\hline
\end{tabular}

We can assume that different cultural activities are closer to specific occupations-as occupation strongly correlates with status, power, and money. A senator might also play bowling but has a higher probability of going to the opera or the theater than a typist.

\section{Example 2}

Half of the company's DATA_SCIENTISTS graduated from Ivy League schools.

The plan of the WAITRESS was to attend evening school next year.

The above-described situation is the same in the second example. Usually, a waitress does not graduate from an Ivy League school, and data scientists do not attend evening schools.

Above the intuitive understanding of these examples, we tested them on our data. We tested the closeness of occupations to certain activities with the cosine similarity of the occupation and the activity words. In the $\mathrm{CC}$ vector space, the cosine similarity of the occupation senator with the word theatre is 0.21 , the same measure for the typist is 0.12 . For bowling, the senator's cosine similarity is 0.05 , but the typist's value is 0.16 . Thus, the senator is closer to the high-end cultural activity, while the typist is closer to the more popular one. These results strengthen the intuitive assumption, namely that different occupations have different likelihoods in these contexts.

At the same time, it is essential to emphasize the different logic of word embedding similarity and similarities of occupational hierarchies created by social scientists. Table 2 presents two occupational pairs as examples. The ISEI distance of the two occupations in the same row is 0 in both cases, meaning these occupation pairs have the same prestige positions. However, the cosine similarity of these occupation pairs is different in the first and second row, which suggests that distances are different in the case of the first and the second row. The reason for this difference lies in the semantic relation of these pairs. In the first row, the two occupations are different in many aspects, despite having the same prestige, while the two occupations in the second row are about identical. Thus, we don't assume to get the same results from the word embedding analysis, as from the different occupational scales, like ISEI.

\section{Results}

\subsection{Common Crawl}

First, we present the results from the Common Crawl corpus. From the list of occupations, the doctor was the most frequent item. Overall, it was the 1496th most frequent word in the list of words contained by the corpus. Driver, writer, cook, judge, editor, lawyer, professor, or attorney were frequent. We can observe a pattern, that those occupations are more frequent in this corpus, which have higher prestige. The Spearman rank correlation between frequency and ISEI score was significant but relatively weak, namely 0.17.

As we have mentioned above, first, we calculated the cosine similarity of the 204 occupations, which were in the most frequent 200,000 words of the vector space. Then we 
used this similarity matrix as an input to extract factors, based on which we detected the main structural dimensions of the occupational semantic space. We tested the model for a different number of factors. In the case of the two-factor solution, the Root Mean Square Residual (RMSR) was 0.07. The explained variances of the two factors were quite similar. In the case of both dimensions, knowledge is an essential factor. Based on the occupations with the highest loading on a given factor, the first dimension is closer to the media domain (e.g., commentator, editor). The second is more relative to the domain of science. (See Table A1 in the Appendix for more details and information on the highest loadings.) We calculated the correlation of the factor loadings with the ISEI scale. ${ }^{5}$ The Pearson correlation was 0.64 in the first dimension and 0.79 in the second dimension-which is relatively high, especially in the second case. The correlation between the frequency of the words and the factor scores was much weaker (below 0.2). We also calculated the partial correlation of factor scores and ISEI with control for the frequency ${ }^{6}$ of occupations, and the correlation values remained the same. These results suggest that both dimensions reflect the vertical positions of occupations, and the frequency of words in the corpus does not interfere with the results.

In the case of a three-factor solution, the RMSR value was 0.06 . The importance of the dimensions was not as equal as in the previous model with two factors. The first factor has the largest Pearson correlation with the ISEI prestige scores $(r=0.71)$. The correlations of the second and third dimensions were moderately high, 0.59 and 0.45 , respectively. High correlation with ISEI means that the corpus contains a strong footprint of the hierarchal social structure.

We have also tested the correlation of the factors of the two- and three-factor models. We found that the correlation of the first factors of the two- and the three-factor solution was 0.9 , and the correlation between the second factors was the same.

Table 3 shows the occupations with the highest and lowest factor loadings on a given factor of the three-factor model. Interpreting the three factors, we found that the first two factors were quite similar but with some critical differences. In the first factor, institutional power seems to be more critical-the chancellor or the dean are good examples. The second factor is structured more based on knowledge and educational level associated with the occupations, while the third factor is built up by the dimensions of the power levels and organizational capacities of the occupations.

For a deeper understanding of the results, we further analyzed the first dimension of the three-factor solution. In the rest of the paper, we refer to this dimension as Occupation Semantic Position Scale (OSPS). The OSPS values are factor loadings, so they are theoretically between -1 and +1 values, but they are above zero in most cases. We can interpret factor scores as correlations, which means that a score above 0.4 or 0.5 is considered to be a high value on the scale.

We calculated whether they are in the same rank order in the two scales for all pairs of occupations. This calculation shows that in 75 percent of the occupation pairs, the or-

\footnotetext{
${ }^{5} \mathrm{~A}$ usual way to create a factor model is to start from a raw data source, calculate the covariance/correlation matrix and then calculate the factor loadings and estimate the factor scores based on these loadings. In this paper, we start from a similarity matrix and calculate the factor loadings. As we do not have raw data here, we could not calculate the factor scores. That has one important implication. Rotated factor scores are statistically independent, but factor scores are not. That is why we have a strong correlation between the extracted factors.

${ }^{6}$ The pre-trained vector space was initially sorted by the frequency of words in the original corpus. So the most frequent word is the first word, the second most frequent is the second, etc. Thus, one has a frequency order for all of the selected occupations. We used this number in the partial correlation calculation as the control (conditioning) variable.
} 


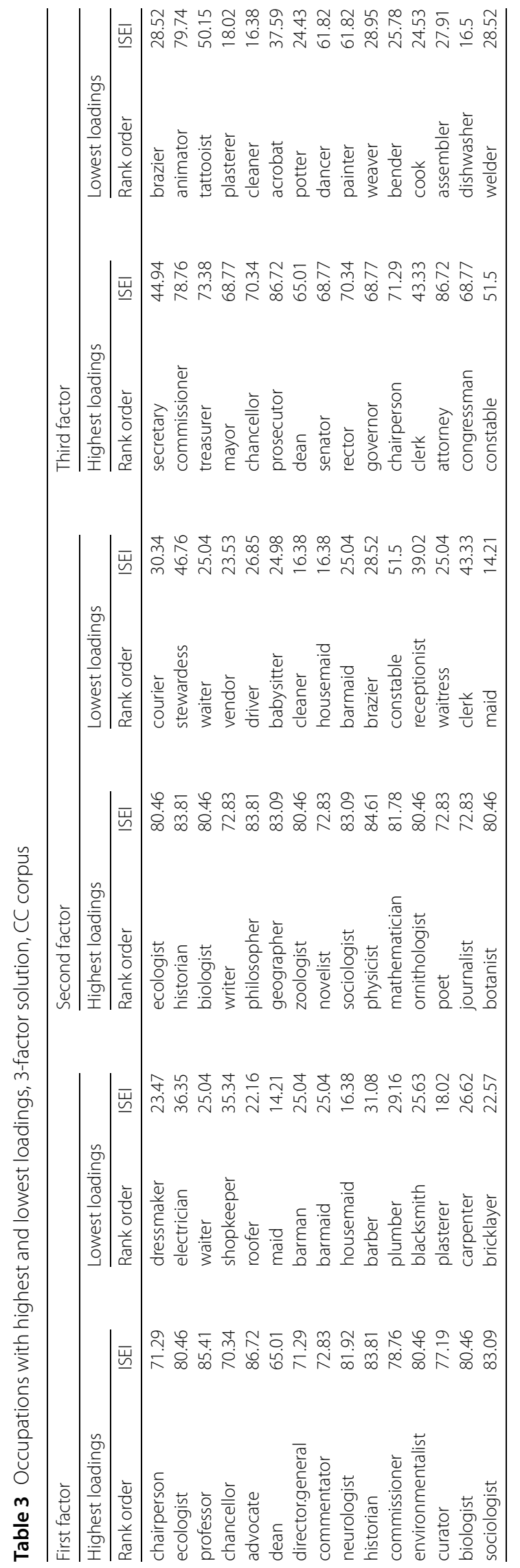




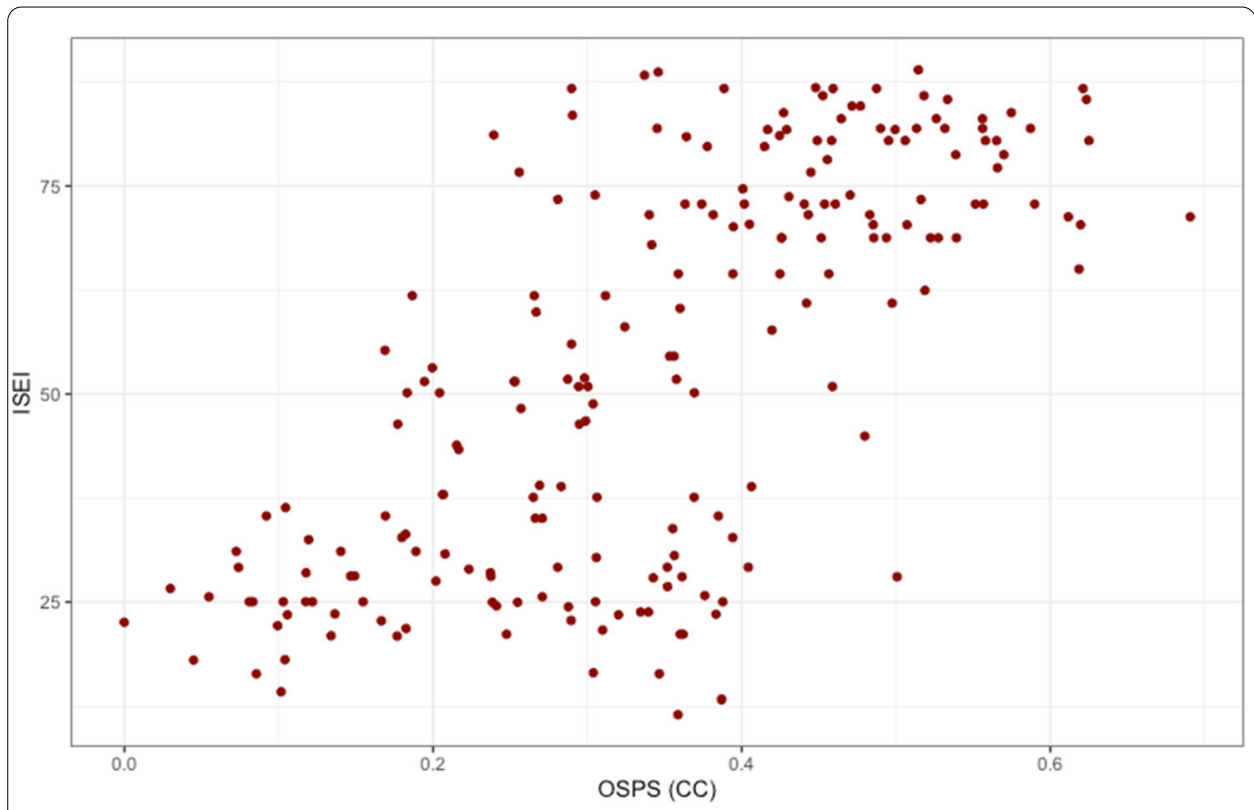

Figure 2 Scatterplot of word embedding based occupation prestige score (Occupation Semantic Position Scale-OSPS) from the CC vector space and ISEI

der was the same. Thus, we can assume that the proximity of occupations in the online texts strongly correlates with the expected educational level and the average income of the selected occupation, which are the basic dimensions of the ISEI prestige score.

We have to emphasize that word embedding method is an unsupervised one, which means that the researchers do not put external information into the model. We haven't used the ISEI prestige scores as an input of the model, nor did we optimized varimax rotation for that. Thus, these results are only based on the information contained in the online texts.

Figure 2 shows the scatterplot of the ISEI and the OSPS scales. The chart also confirmed the high correlations between the two scores (Pearson correlation coefficient was 0.71 and Spearman correlation coefficient was 0.69 ), but at the same time, we have found remarkable differences. Some occupations like doctor, dentist, pharmacist, or solicitor were positioned relatively low in the OSPS while high on the ISEI. One possible explanation could be that the position of an occupation on the OSPS depends not only on the prestige of the occupation but also on other dimensions, like the reflection of the domain, which surrounds the occupation. For example, being a dentist is a high prestige job, paired with a high educational level and high income, but (1) being sick is not a favorable situation (which feelings can be mirrored in the texts) and (2) everybody can be sick, irrespective of their social status: health care professionals provide services to the general public, which means they have links to all levels of the social structure. As health-related occupations are all affected by these circumstances, this can be one reason that they are scored lower. Nevertheless, further qualitative analysis is needed for a deeper understanding of these differences.

Knowledge and power level are essential factors of prestige, so it is not surprising we found these dimensions behind the hierarchical structure of semantic positions of occupations. At the same time, the wage doesn't appear as an organizing principle in this hier- 
archy; however, it is an important dimension of the prestige scales. We wanted to know if wage can be detected as a background dimension in further factors, so we created 4- and 5 -factor models.

In the case of the 4-factor model, the first three dimensions were quite similar to those found in the 3-factor solution. The primary structuring dimension of the fourth factor was gender: occupations with the five highest loadings were receptionist, waitress, babysitter, manicurist, and hairdresser. In the 5-factor model, we still haven't detected wage as an organizing dimension of any factors. We have found that health-related occupations score high on the fifth dimension-like a domain-specific one. We could also observe that as we increase the number of factors in the model, the correlation of the first factor with the ISEI becomes lower and lower.

\subsection{Wikinews}

To test the robustness of our results, we repeated our analysis on a different corpus, namely on the Wikinews corpus. In this corpus, the most frequent occupation was the editor, but judge, politician, or lawyer was also regular, such as journalist, writer, and singer. Most of these are higher prestige occupations, which are related to the domains of politics, media, and culture. For comparison, we run the same factor analyses as on the CC-based embedding. The results were more similar than we expected. In the case of the 3 -factor solution, the Pearson correlations of the first factor scores of the two corpora were 0.97, the correlation of the second factor was 0.93 , and of the third factor, it was 0.82 . These results suggest that the factors in the two corpora show a similar structure of the occupations.

With a more detailed qualitative analysis, we could find minor differences between the first factors of the Wikinews and CC corpus. Some manual-labor occupations like locksmith and dishwasher got higher scores in the Wikinews corpus. Some literature and artrelated occupations, like poet, novelist, composer, or painter, scored higher in the CC corpus. Table 4 presents the occupations with the highest loadings in each dimension.

The interpretation of the first three dimensions is quite similar to the ones in the CC corpus. The first factor shows a mixed organizing pattern built of power and knowledge. In the case of the second factor, the science-related occupations scores high. The dimension behind the third factor is about power level and organizational capacity. The Pearson correlation of the first dimension with the ISEI score was 0.71 (see Fig. A1). We found that 74 percent of the occupation pairs are in the same order in the Wikinews-based first factor and the ISEI scale. In addition to the similarities, we also found differences: some animal- and farm-related occupations (e.g., breeder, fisher, planter) score much higher on the semantic scale, and some health-related occupations (e.g., doctor, surgeon, dentist, pharmacist) score higher on the ISEI scale.

We have also tested the 4- and 5-factor solutions here. Similar to the result of the CC corpus, the 4th factor can be interpreted as the gender dimension: occupations like a nanny, hairdresser, receptionist, babysitter, or waitress score high there. Just as in the CC corpus, the 5 th dimension was a domain-related one. However, it is interesting that in the current (Wikinews) corpus, it was not the health domain, which characterized the scale, but the domain of media and culture, with highly scored occupations like novelist, poet, singer, composer, etc. dramatist, lyricist, or writer. 


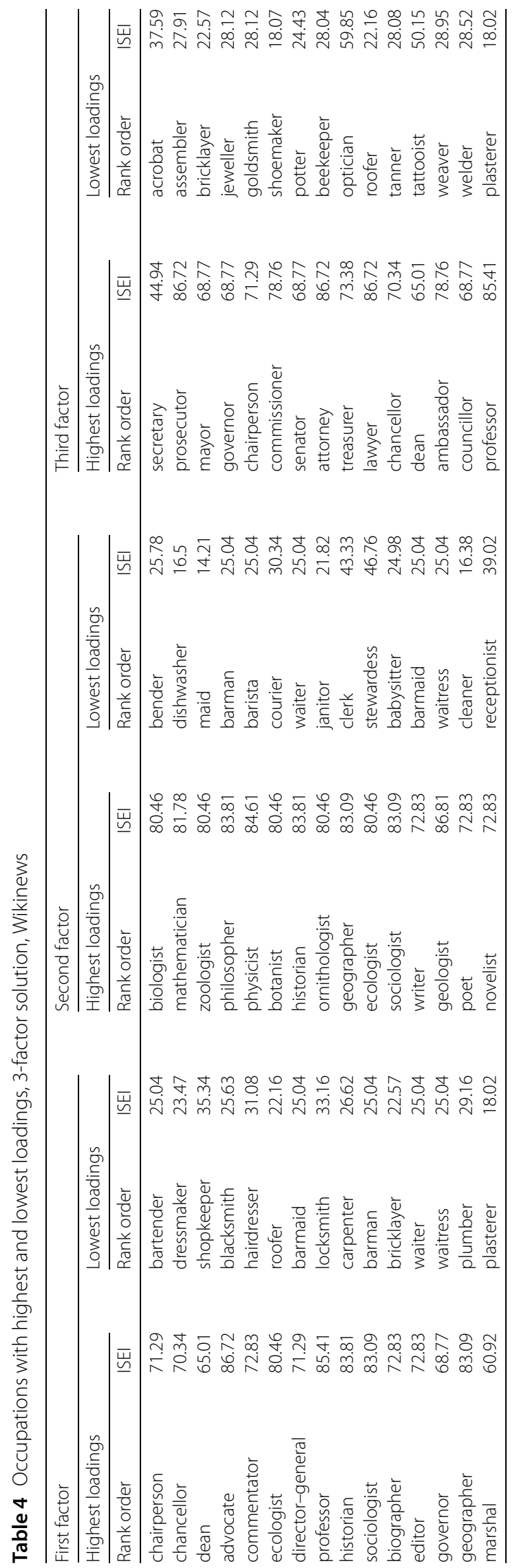




\subsection{Wikinews with sub-word information}

The last word embedding we tested was also built on the Wikinews corpus, but the training phase of this model also took into account sub-word information. With this solution, partly identical words or words with the same root are closer in the vector space. The same 3-factor solution was applied here, and the interpretation of the three factors is the same as in the previous cases. (For more details about these factors, see Table A2 in the Appendix).

The interpretation of the factors showed that institutional power is an essential aspect in the first factor, but knowledge also matters there. The second factor was related to the knowledge and educational level associated with the occupations. In contrast, the third factor was scaled on the power levels and organizational capacities of the occupations. This later factor is close to the domain of politics.

The Pearson correlation of the first dimension with the ISEI score was 0.78 . According to the rank order, 77 percent of the occupation pairs were the same on both scales, namely in the first factor of this corpus and the ISEI. The occupations, which are much higher on the semantic scale are rancher, planter, and astrologist. Other occupations are underestimated compared to the ISEI: such as in the case of the CC corpus; these are domain-specific occupations. Some are health-related occupations, such as dentist, doctor, pharmacist, and surgeon; some are financial occupations, like banker or accountant; and some are judicial systems related occupations like judge, lawyer, or solicitor.

We also tested the 4- and 5-factor solution here. The 4th factor showed the gender dimension again with high scores at occupations like nanny, hairdresser, receptionist, babysitter, and waitress. The 5th factor was again a domain-related one, namely the domain of media and culture with high scores at occupations like novelist, poet, singer, composer, dramatist, lyricist, and writer-just like in the case of the Wikinews corpus.

\subsection{Robustness-stability of occupational positions in different vector spaces}

The correlation of the factor loadings across different embeddings seems to be strong. The Pearson correlations of the first factor scores of the CC and Wikinews embeddings was 0.97 , between the second factors it was 0.93 , and between the third factors 0.82 . These results provide strong evidence for the robustness of the results and implicate that occupation positions are pretty stable across different corpora.

To further test this stability, we wanted to compare the positions of the occupations in the different vector spaces. To do this, we had to align the Wikinews vector space to the $\mathrm{CC}$ vector space with Procrustes rotation the way we described earlier. As we stated above, in this aligned vector space, the cosine similarities of the words are the same as before the alignment. Still, we can calculate the similarity of the same occupation between the two vector spaces. The average similarity of the occupations between the two corpora was 0.79 . There is no clear threshold of what similarity level can be interpreted as 'strong', but we can observe that only close concepts have a similarity value around 0.7. An intuitive example for this in the $\mathrm{CC}$ embedding is dog breeds, like Labrador and Beagle, which have a similarity value of around 0.7. To see the implications of the alignment, we calculated the closeness of every occupation in the rotated (aligned) corpus with itself in the original (non-rotated) corpus. Further, the closeness values between each occupation in the initial corpus and the other occupations in the rotated corpus were determined. As mentioned 
before, the average of the first closeness value was 0.79 , while the second measure's average was 0.29 , and the minimum pairwise distance was 0.35 . We also calculated the distance of specific occupations with the 20 closest words in the original CC corpus. We computed the same value (with the same occupations and words) in the aligned matrix. The first value on average was 0.59 ; the second was 0.52 . We also calculated the distance of specific occupations and all other words in the $\mathrm{CC}$ and the aligned vector space. The average distance was 0.11 in the $C C$ embedding and 0.13 in the aligned vector space. Thus, the proximity of words remains similar after the rotation. These results confirm the robustness of the alignment approach.

Although the average similarity measure implicates high stability between the embeddings, there are some occupations where we found lower-but in absolute values still high-similarities. Occupations with the lowest similarity scores (between 0.65 and 0.7) were the following: masseur, dishwasher, rheumatologist, manicurist, zookeeper, editor, bender, locksmith, dentist, and tanner. We cannot observe a clear organizing principle, but some of these occupations are pretty rare now, like the tanner or the bender.

We calculated the Pearson correlation coefficient of Wikinews frequency of occupations and the stability measure, which was 0.59 . This result is parallel with earlier findings, namely that those words are stable across time, which are frequent (Hamilton-LeskovecJurafsky [16]). Our results show that it is applicable not only for temporal analysis but also for the analysis of different corpora (and embeddings) created approximately simultaneously. Stability also positively correlated with the ISEI score (Pearson $r=0.36, p=0.00$ ). The direction of the correlation suggests that the positions of more prestigious occupations are more stable across corpora. Still, this result should be treated with caution, as this effect partly exists because more prestigious occupations are also more frequent (at least in the two corpora we used). However, even after controlling for the frequencies of the words, the correlation remains significant (Pearson $r=0.19, p=0.000$ ) between ISEI score and stability.

\section{Discussion}

We raised two questions about the usefulness of word embedding-based semantic analysis related to the description of occupational structure in particular occupational rankings. Are the results comparable with standard results, and is it possible to gain additional insights about the social positions of occupations? Both questions raised at the beginning of the paper have been given affirmative answers. The results show the fundamental similarity of the social structure obtained from text analysis to the structure described by Ganzeboom and Treiman [12]. But a more detailed analysis also reveals some differences.

Our paper focused more on methodological aspects, and we put less emphasis on the substantive analysis of the results. But the first-superficial-analysis revealed an exciting dimension of the occupation structure: the power and organizational aspect. As far as we know, the importance of this factor is not discussed in the mainline of stratification literature in sociology.

It has been widely discussed (Johnson [18]) that power is a crucial component of the prestige of an occupation. But our results indicate the interplay between knowledge and organizational capacity. In the 3 -factor solution, each is characterized by the presence 
of one or both of these, and power presents itself as a combination of knowledge and organizational capacity. It is not a surprise that knowledge, also in itself, is a fundamental dimension, but it does seem entirely novel that organizational capacity, also in itself, is a contributing dimension. Freidson [11] distinguishes two types of elites: knowledge and administrative elites in his classic work. Waring [34] re-appraised the Freidson model and added two extra elite types, corporate and government elite. Our third factor mirrors the importance of this governance elite as a vital factor that structures the occupational space.

The results proved relatively stable, as repeating the analyses on two different corpora yielded strongly similar results. Correlations of the factors between the two corpora were high and substantively significant. After the alignment of the second corpus on the first one, we found strong similarities in the positions of the occupations across corpora. Although we don't have data for measuring other stability indicators, we know from other studies (Hamilton-Leskovec-Jurafsky [16]) that concept stability is lower for words, which are frequently used in different environments-that is called polysemy in linguistic. It is also known that the position of a concept changes over time (Kozlowski et al. [21]), so further analysis may also take into account the period during which the original corpora were collected.

The results were also stable from a choice of method perspective. We tested many approaches like Pearson correlation instead of cosine similarity or different factor methods and rotation techniques, and we could only observe minor differences at the end. So the extracted structure is very robust in many ways.

We decided to use pre-trained corpora in this paper and not trained unique word embeddings. These pre-trained corpora are available for everyone, so it is easy to reproduce our results and make further steps in this area. One shortcoming of this approach is that we could not narrow the geographical focus of the results, and we could not influence what type of texts are included in the training set. However, previous studies showed (Treimann [33]) that prestige scores are highly correlated in developed countries. So our general approach might not lead to significant biases. The fact also confirms the validity of the results, that results from different corpora and word embedding was similar.

Nevertheless, it could be logical to repeat this analysis with self-trained wordembeddings, where we have more substantial control of the selected texts. Training our models has a further advantage; we could pre-process the texts before calculating the vector spaces. For social science analysis, pre-processed texts could work better as the information is focused here, and there is less noise in those texts. We could also add bigrams to the model, which might be essential to catching the two-word length occupations like "social scientist." Further studies are needed to understand how pre-preprocessing influences word embedding features and how this affects any social science-related analysis.

Our paper presents exploratory research using textual data, with fairly new methods in the social sciences. However, it has already been demonstrated that unsupervised learning methods such as the analysis of word embeddings can find interesting patterns and generate new hypotheses (Nelson [25]). Both qualitative and quantitative approaches are needed to exploit this potential in understanding societies fully. 


\section{Appendix}

\section{A.1 List of occupations}

accompanist, accountant, acrobat, actor, actuary, admiral, advocate, agriculturist, agrologist, agronomist, allergist, ambassador, anaesthesiologist, anatomist, animator, appraiser, archaeologist, architect, assembler, astrologer, astronaut, athlete, attorney, auditor, babysitter, baker, ballerina, banker, barber, barista, barkeeper, barmaid, barman, bartender, beekeeper, bender, biographer, biologist, bishop, blacksmith, blocklayer, boatman, bodyguard, bookkeeper, bookmaker, botanist, boxer, brazier, breeder, brewer, bricklayer, broker, butcher, cardiologist, carer, carpenter, cellist, ceo, chairperson, chancellor, chaplain, chef, chemist, cleaner, clerk, coalman, coastguard, coder, comedian, commentator, commissioner, composer, congressman, congresswoman, constable, cook, copywriter, coroner, corporal, councillor, courier, curator, dancer, dean, dentist, director-general, dishwasher, dockmaster, doctor, doorkeeper, dramatist, dressmaker, driller, driver, dustman, ecologist, editor, electrician, environmentalist, etcher, farmer, firefighter, fireman, fisher, flamecutter, footballer, forger, friar, furrier, gaoler, gardener, geodesist, geographer, geologist, goatherd, goldsmith, governor, grazier, grocer, hairdresser, head-teacher, historian, hooker, providing sexual services, housemaid, innkeeper, janitor, jeweller, journalist, judge, juggler, lawyer, lecturer, librarian, locksmith, lyricist, macroeconomist, maid, managing-director, manicurist, marketer, marshal, masseur, mathematician, mayor, mechanic, meteorologist, midwife, miner, money-lender, monk, nanny, neurologist, nightwatchman, novelist, nurse, optician, ornithologist, painter, paratrooper, parliamentarian, pastry-cook, pharmacist, philosopher, photographer, physicist, physiotherapist, planter, plasterer, plumber, poet, policeman, policewoman, politician, postman, postmaster, potter, priest, professor, programmer, proofreader, prosecutor, prostitute, psychiatrist, psychologist, psychotherapist, publicist, rabbi, radiographer, rancher, receptionist, rector, retailer, rheumatologist, roofer, sailor, secretary, senator, setter-operator, shepherd, shoe-polisher, shoemaker, shopkeeper, signwriter, singer, sociologist, soldier, solicitor, sommelier, sous-chef, stationmaster, statistician, steward, stewardess, stonecutter, storekeeper, surgeon, tailor, tanner, tattooist, telemarketer, telephonist, tiler, translator, treasurer, typist, vendor, waiter, waitress, weaver, webmaster, welder, writer, zookeeper, zoologist

Table A1 Occupations with highest loadings, 2-factor solution, CC

\begin{tabular}{ll}
\hline Factor 1 & Factor 2 \\
\hline curator & historian \\
editor & biologist \\
geographer & zoologist \\
professor & sociologist \\
sociologist & geographer \\
biologist & physicist \\
chairperson & journalist \\
historian & ornithologist \\
environmentalist & lecturer \\
commentator & writer \\
\hline
\end{tabular}


Table A2 Occupations with highest loadings, 3-factor solution, Wikinews_subwords

\begin{tabular}{lll}
\hline Factor 1 & Factor 2 & Factor 3 \\
\hline professor & biologist & commissioner \\
congresswoman & zoologist & secretary \\
biographer & ecologist & mayor \\
CEO & physicist & chancellor \\
ecologist & ornithologist & chairperson \\
neurologist & sociologist & prosecutor \\
director-general & mathematician & governor \\
chairperson & geographer & senator \\
chancellor & botanist & attorney \\
dean & geologist & treasurer \\
\hline
\end{tabular}

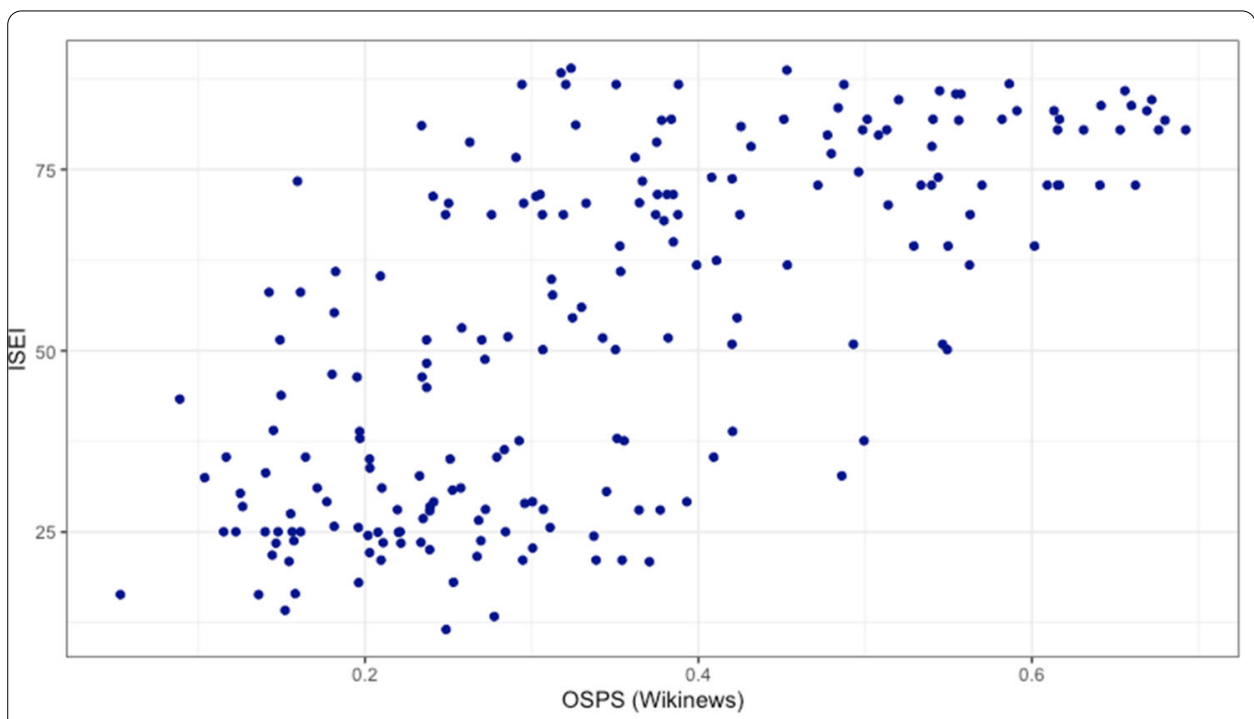

Figure A1 Scatterplot of word embedding based occupation prestige score (Occupation Semantic Position Scale-OSPS) from the CC vector space and ISEI

\section{Acknowledgements}

The authors would like to thank the reviewers for their substantial comments and suggestions.

\section{Funding}

The work of Zoltan Kmetty was funded by the Premium Postdoctoral Grant of the Hungarian Academy of Sciences. The work of Julia Koltai was funded by the Premium Postdoctoral Grant of the Hungarian Academy of Sciences.

\section{Abbreviations}

NLP, Natural Language Processing; ISEl, International Socio-Economic Index; SIOPS, Standard International Occupational Prestige Scale; CC, Common Crawl; PCA, Principal Component Analysis; RMSR, Average residuals for the similarity matrix: OSPS, Occupation Semantic Position Scale.

\section{Availability of data and materials}

The pre-trained word vectors are available here: Common Crawl: http://commoncrawl.org. Wikinews:

https://fasttext.cc/docs/en/english-vectors.html.

\section{Declarations}

\section{Competing interests}

The authors declare that they have no competing interests.

\section{Authors' contributions}

ZK: Concept, computations, analysis, discussion. JK: Concept, theoretical part, discussion. TR: Concept, theoretical part, discussion. All authors read and approved the final manuscript. 


\section{Author details}

'CSS-Recens Research Group, Centre for Social Sciences - Hungarian Academy of Sciences Centre of Excellence, Tóth Kálmán u. 4, 1097, Budapest, Hungary. ²Faculty of Social Sciences, Eötvös Loránd University, Pázmány Péter sétány 1/A, 1117, Budapest, Hungary. ${ }^{3}$ Department of Network and Data Science, Central European University, Vienna, A-1100, Austria.

\section{Publisher's Note}

Springer Nature remains neutral with regard to jurisdictional claims in published maps and institutional affiliations.

Received: 16 May 2021 Accepted: 15 November 2021 Published online: 27 November 2021

\section{References}

1. Bales RF (1950) A set of categories for the analysis of small group interaction. Am Sociol Rev 15(2):257-263

2. Bolukbasi T, Chang KW, Zou JY, Saligrama V, Kalai AT (2016) Man is to computer programmer as woman is to homemaker? Debiasing word embeddings. In: Advances in neural information processing systems, pp 4349-4357

3. Bukodi E, Dex S, Goldthorpe JH (2011) The conceptualisation and measurement of occupational hierarchies: a review, a proposal and some illustrative analyses. Qual Quant 45(3):623-639

4. Caliskan A, Bryson JJ, Narayanan A (2017) Semantics derived automatically from language corpora contain human-like biases. Science 356(6334):183-186

5. Chan TW, Goldthorpe JH (2004) Is there a status order in contemporary British society? Evidence from the occupational structure of friendship. Eur Sociol Rev 20(5):383-401

6. Connelly R, Gayle V, Lambert PS (2016) A review of occupation-based social classifications for social survey research. Methodol Innov 9:2059799116638003

7. Devlin J, Chang MW, Lee K, Toutanova K (2018) Bert: pre-training of deep bidirectional transformers for language understanding. arXiv preprint. 1810.04805

8. Erikson R, Goldthorpe JH, Portocarero L (1979) Intergenerational class mobility in three western European societies: England, France and Sweden. Br J Sociol 30(4):415-441

9. Evans JA, Aceves P (2016) Machine translation: mining text for social theory. Annu Rev Sociol 42:21-50

10. Firth JR (1957) A synopsis of linguistic theory. Studies in linguis-tic analysis. Blackwell, Oxford

11. Freidson E (1984) The changing nature of professional control. Annu Rev Sociol 10(1):1-20

12. Ganzeboom HB, Treiman DJ (1996) Internationally comparable measures of occupational status for the 1988 international standard classification of occupations. Soc Sci Res 25(3):201-239

13. Garg N, Schiebinger L, Jurafsky D, Zou J (2018) Word embeddings quantify 100 years of gender and ethnic stereotypes. Proc Natl Acad Sci 115(16):E3635-E3644

14. Goldthorpe JH, Hope K (1972) Occupational grading and occupational prestige. Soc Sci Inf 11(5):17-73

15. Goldthorpe $\mathrm{JH}$, Halsey AH, Heath AF, Ridge JM, Bloom L, Jones FL (1982) Social mobility and class structure in modern Britain

16. Hamilton WL, Leskovec J, Jurafsky D (2016) Diachronic word embeddings reveal statistical laws of semantic change. arXiv preprint. 1605.09096

17. Hays DC (1960) Automatic content analysis. Rand Corp., Santa Monica

18. Johnson TJ (2016) Professions and power (Routledge revivals). Routledge

19. Joseph K, Morgan JH (2020) When do word embeddings accurately reflect surveys on our beliefs about people? arXiv preprint. 2004.12043

20. Joulin A, Grave E, Bojanowski P, Mikolov T (2016) Bag of tricks for efficient text classification. arXiv preprint. 1607.01759

21. Kozlowski AC, Taddy M, Evans JA (2019) The geometry of culture: analyzing the meanings of class through word embeddings. Am Sociol Rev 84(5):905-949

22. Lazer D, Radford J (2017) Data ex machina: introduction to big data. Annu Rev Sociol 43(1):19-39

23. Meraviglia C, Ganzeboom HB, De Luca D (2016) A new international measure of social stratification. Contemp Soc Sci $11(2-3): 125-153$

24. Mikolov T, Chen K, Corrado G, Dean J (2013) Efficient estimation of word representations in vector space. arXiv preprint. 1301.3781

25. Nelson LK (2020) Computational grounded theory: a methodological framework. Sociol Methods Res 49(1):3-42

26. Németh R, Koltai J (2021) The potential of automated text analytics in social knowledge building. In: Rudas T, Péli G (eds) Pathways between social science and computational social science-therories, methods and interpretations. Springer, New York

27. Pennington J, Socher R, Manning CD (2014). GloVe: Global Vectors for Word Representation

28. Prandy K, Lambert P (2003) Marriage, social distance and the social space: an alternative derivation and validation of the Cambridge scale. Sociology 37(3):397-411

29. Revelle W (2018) psych: procedures for personality and psychological research. Northwestern University, Evanston. https://CRAN.R-project.org/package=psych. Version 1.8.12

30. Rose D, Harrison E (2007) The European socio-economic classification: a new social class schema for comparative European research. Eur Soc 9(3):459-490

31. Savage M, Burrows R (2007) The coming crisis of empirical sociology. Sociol: J Brit Sociol Assoc 41:885-899

32. Schakel AM, Wilson BJ (2015) Measuring word significance using distributed representations of words. arXiv preprint. 1508.02297

33. Treiman DJ (1977) Occupational prestige in comparative perspective. Academic Press, New York

34. Waring J (2014) Restratification, hybridity and professional elites: questions of power, identity and relational contingency at the points of 'professional-organisational intersection'. Sociol Compass 8(6):688-704 